\title{
Neuropsicologia, formação e desafios
}

\author{
Neuropsychology, training and challenges
}

\author{
Diogo Fagundes Pereira ${ }^{1}$ \\ 'Faculdade Arthur Sá Earp Neto (FASE), Petrópolis, RJ, Brasil.
}

Recebido em: 21/04/2017 / Aceito em: 26/09/2017 / Publicado em: 01/10/2017

diogofagundes.psi@gmail.com

\section{RESUMO}

O presente trabalho trata-se de uma pesquisa documental, no qual foram analisados currículos de cursos de pós-graduação em Neuropsicologia no estado do Rio de Janeiro. Embora essa especialidade não seja exclusiva do domínio da psicologia, existem diferenças significativas nos programas de pós-graduação nessa área, tanto em relação à carga horária, como na distribuição das disciplinas. Objetivo: identificar as principais diferenças encontradas nas ementas dos cursos e verificar as implicações dessa formação para a prática profissional. Método: realizou-se uma pesquisa documental no dispositivo de busca Google, com as palavras-chave: "Pós-graduação, Neuropsicologia, Rio de Janeiro". Resultados: foram encontrados 7 programas que foram divididos e analisados nas categorias: carga horária, ênfases, estágios e público-alvo. Os resultados apontaram que não existe regularidade nas cargas horárias, bem como, nas escolhas das disciplinas. Considerações finais: não existem critérios definidos das ênfases: educação, avaliação e reabilitação neuropsicológica e aponta ainda, a necessidade de estruturar o programa dessa especialidade, pois independente da sua formação, o profissional estará habilitado em trabalhar com a neuropsicologia em todo território nacional, o que poderia implicar em prejuízos na saúde e bem estar dos indivíduos, assim como, numa estagnação no desenvolvimento das pesquisas em neurociências.

Palavras-Chave: Neuropsicologia; Currículo; For-

\section{ABSTRACT}

The present study is a documentary research in which curriculum of postgraduate courses in Neuropsychology in the state of Rio de Janeiro were analyzed. Although this specialty is not unique to the field of psychology, there are significant differences in postgraduate programs in this area, both in relation to the workload and in the disciplines distribution. Objective: to identify the main differences found in the course syllabus and to verify the implications of this training for professional practice. Method: a documentary search was performed on the Google search device with the keywords: "Post-graduation, Neuropsychology, and Rio de Janeiro". Results: 7 programs were found that were divided and analyzed in the categories: workload, emphases, stages and target audience. The results showed that there is no regularity in the schedules, as well as in the disciplines choice. Closing remarks: there are no defined criteria of the emphases: education, evaluation and neuropsychological rehabilitation and there is also the need to structure the program of this specialty, since regardless of their training, the professional will be able to work with neuropsychology throughout the national territory. This could lead to impairments in the health and well-being of individuals, as well as stagnation in the development of neuroscience research.

Keywords: Neuropsychology; Curriculum; Training. mação. 


\section{INTRODUÇÃO}

Esse estudo nasceu de uma necessidade embrionária de investigar as diversas formações em neuropsicologia. Embora a titulação seja a mesma em todo país, o fazer neuropsicológico, na prática, acaba chegando às vezes, até o seu processo de avaliação, e quando avança para a reabilitação, esse fazer se torna segmentado e diverso.

Desvelar o conhecimento cerebral e suas relações no comportamento, sempre despertou muita curiosidade nos homens, assim como, conhecer a fundo essa "caixa preta" sempre foi um grande desafio. A ciência, antes do século XIX, estabeleceu diferenças significativas entre os transtornos que eram de origem orgânica e psicológica e isso sempre gerou um conhecimento dicotômico, resultando em técnicas e procedimentos de bases epistemológicas distintas. Com Philippe Pinel (1745-1826), psiquiatra francês, os transtornos mentais começaram e ser vistos com outras perspectivas; saiu de uma visão estritamente biológica e inaugurou uma visão psicogênica dos transtornos mentais, fortalecendo por exemplo, a ciência psicológica. No secular desejo de combinar esses conhecimentos: comportamentos, emoções e suas relações com os substratos neurais, que foi configurando uma nova ciência: a neuropsicologia.

Em seguida, esse saber foi reconhecido como especialidade dentro da psicologia. E, como o Conselho Federal de Psicologia (CFP) entende esse conhecimento, principalmente, como integrado na prática clínica e na pesquisa. Associou-se também à resolução do MEC que trata da legalização das pós-graduações no país, o que possibilitou as análises propostas nesse estudo.

A ideia central desse estudo repousa no fato da diversidade curricular dessa especialidade impactar na atuação prática desses profissionais. Um pedagogo que fizer a formação em neuropsicologia poderá encontrar dificuldade de fazer, por exemplo, um rastreio cognitivo das principais funções mentais, ou mesmo, uma avaliação neuropsicológica completa, pelo fato dos principais instrumentos desse domínio, serem de uso exclusivo de psicólogo.

Com esse delineamento teórico, objetiva-se identificar a as principais diferenças encontradas nas ementas dos cursos e verificar as implicações dessa formação para a prática profissional.

\section{Fundamentação teórica}

É antigo o desejo do homem em compreender o funcionamento cerebral, seus processos mentais e psicopatologias. Desde antiguidade, já ouvia-se falar do interesse em correlacionar esse binômio mente e cérebro. A ciência, durante muito tempo, polarizou esse conhecimento, deixando cada domínio em seu lugar, de maneira que até meados do século XIX, a etiologia dos transtornos mentais eram de base orgânica, até o surgimento do Psiquiatra Francês Philippe Pinel, autor de transição no entendimento dos transtornos mentais, que abriu espaços para discutir as origens psicogênicas desses transtornos. É quando, no final do século XIX e início do $X X$, algumas pesquisas já sinalizavam o interesse de combinar mente e cérebro em suas variáveis de estudo. ${ }^{1}$ Em 1913 surge o termo "Neuropsicologia", sendo proferido pela primeira vez em uma conferência nos Estados Unidos, por William Osler, aparecendo mais tarde, em 1949, como como um subtítulo na obra de
Donald Hebb chamada "The Organization of Behavior: A Neuropsychological Theory". ${ }^{2}$ Esse novo campo do saber, que integra conhecimento dos processos mentais e bases biológicas, é constituído por estudar as expressões comportamentais das disfunções cerebrais. ${ }^{3}$

Três grandes abordagens metodológicas chamaram atenção no estudo das raízes à Neuropsicologia. A primeira escola clássica foi embasada em estudos de casos clínicos, com ênfases em correlações anátomo-clínica; a segunda teve mais um aspecto psicométrico, com ênfase nos dados quantitativos e por fim; a terceira, considerada escola experimental, focou em caso clínico único e atividades experimentais. É possível que essas escolas, tenham buscado ultrapassar a tradição post mortem, dado aos estudos do cérebro da antiguidade.

A neuropsicologia utiliza-se de um método que, a partir da alteração de alguma função cognitiva ou psicológica, pode chegar a algum tipo de lesão cerebral, ou mesmo uma alteração de atividade cerebral, assim como, a partir de lesões já identificadas, podem ser correlacionadas com sistemas e processos psicológicos. ${ }^{4} \mathrm{Ou}$ seja, a neuropsicologia propõe caminhos que associam aspectos neurológicos e psicológicos, que podem trazer benefícios e bem estar psicológicos para seus usuários. Desse modo, ela tornou-se mais uma via para análise da atividade mental e sua finalidade é possibilitar o entendimento de certas operações cognitivas ou suas disfunções e relacioná-los com os sistemas neurais e seus componentes. ${ }^{5}$

\section{Neuropsicologia enquanto especialidade da psicologia}

Em 2004, o Conselho Federal de Psicologia- CFP reconhece a Neuropsicologia, enquanto especialidade, inclusive para concessão e registro do título de especialista. Esse campo do saber atua no diagnóstico, acompanhamento, tratamento e pesquisa da cognição, personalidade, emoções, comportamento e suas relações com o funcionamento cerebral, utiliza-se para esse processo, conhecimentos da área clínica e das neurociências. ${ }^{6}$

O objetivo teórico da neuropsicologia é ampliar modelos já conhecidos e desenvolver novas hipóteses sobre as interações cérebro e comportamento. Tem como objetivos práticos, levantar dados que permitam diagnosticar, planejar intervenção, reabilitação, no campo da pesquisa. Desenvolve materiais e instrumentos para melhorar a prática e eficiência nos resultados, configurando assim, três campos específicos, a saber: diagnóstico, reabilitação e pesquisa.

O diagnóstico é realizado através de instrumentos, que são desenvolvidos pela própria ciência neuropsicológica ou mesmo por áreas afins como a psicologia cognitiva. Esse campo, o profissional deverá investigar o desempenho das funções cognitivas como memória, atenção, pensamento, funções executivas, linguagem, entre outras. A partir desse processo é possível planejar todas as atividades subsequentes, como reabilitação, estimulação, etc.

Outro campo é a reabilitação, que é secundário à avaliação, onde o processo está vinculado à compensação de danos ou lesões cerebrais, estimulações de áreas que estão com poucos desempenho, enfim, um trabalho direto com as funções cognitivas compro- 
metidas, visando o reestabelecimento e mudança nas capacidades e habilidades do paciente.

Por fim, a pesquisa, que é responsável por avanços nessa área, descobertas de correlações entre comportamentos, funções cognitivas e resposta cerebral. Essa campo do saber neuropsicológico é fundamental para as duas outras atividades anteriores (avaliação e reabilitação).

\section{Legalização da Pós-Graduação}

A resolução $n^{\circ} 1$ de 8 de junho de 2007,, estabelece normas para o funcionamento dos cursos de pós-graduação Lato Sensu. Essas normas são bem gerais e tratam desde a oferta do curso à expedição do seu certificado. De maneira geral, somente as IES (Instituições de Ensino Superior) podem oferecer os cursos de pós-graduação no país, cabendo as mesmas, a responsabilidade do projeto pedagógico, corpo docente, metodologia, etc.

Sobre o corpo docente, exige-se que $50 \%$ seja de mestres e doutores, e os demais especialistas ou com reconhecida capacidade técnico- profissional. Os cursos independem de autorização, reconhecimento e renovação, embora possam ser avaliados quando da recredenciamento da IES. Ademais, exige-se que o curso tenha carga horária mínima de 360 horas, não computadas as horas de estudos individual ou em grupo.

É imperioso demarcar uma diferença entre a graduação a pós-graduação, em relação à autonomia pedagógica e curricular. A primeira, independente do curso, tem uma base nacional comum instituída pelo Ministério da Educação (MEC) e suas ênfases, já na segunda, autonomia fica à cargo da universidade.

\section{MÉTODO}

Trata-se de uma pesquisa documental, método que assemelha-se muito à pesquisa bibliográfica, porém, a diferença está na natureza das fontes. Ela consiste em um exame de diversos materiais que ainda não sofreram nenhum trabalho de análise ou mesmo que pode ser reexaminados objetivando outras interpretações. Pode-se dizer que esse desenho metodológico é aquele que os dados obtidos são estritamente originados de documentos, visando extrair informações, a fim de compreender um fenômeno. ${ }^{8}$

Mediante o recorte da pesquisa, foram inseridas as palavras-chave: "pós-graduação, neuropsicologia, rio de janeiro" no dispositivo de busca Google. Após identificação dos cursos, foi extraído os programas dos sites institucionais das IES e dividido em quatro categorias: Carga horária total do curso; Ênfases, Estágios e Público-Alvo, a categoria ênfase, subdividiu-se em (educação, avaliação e intervenção), no item educação verificou se a organização das disciplinas contemplava apenas a disseminação do saber neuropsicológico; no item avaliação, quais cursos priorizavam o ensinamento do processo de avaliação neuropsicológica e por fim, o item intervenção, analisou-se quais currículos lidavam com a reabilitação.

Analisou-se também a oferta de estágio por esses programas e qual o público alvo, se somente psicólogos ou diplomados de outras áreas do saber, também pode- riam contemplar o perfil do ingressante.

\section{RESULTADOS}

Foram encontrados 07 currículos de pós-graduação em Neuropsicologia no estado do Rio de Janeiro, todos analisados conforme as categorias propostas no delineamento metodológico desse estudo.

\section{Carga horária dos cursos}

Conforme tabela 1, apenas dois programas ofereceram a carga horária mínima estabelecida por lei, que são de 360 horas; os demais estabeleceram carga horária superior. É válido salientar que um curso está disponível em formato EaD - Educação à distância.

Tabela 1 - Carga horária dos cursos.

\begin{tabular}{cc}
\hline Programa & Carga Horária \\
\hline 1 & $495^{*}$ EAD \\
2 & 390 \\
3 & 500 \\
4 & 360 \\
5 & 360 \\
6 & 522 \\
7 & 510 \\
\hline
\end{tabular}

\section{Ênfase do Curso}

Na tabela 2 o programa número 1 restringe o seu conhecimento apenas ao ensino dos conteúdos da neuropsicologia, dissociado de estágios e intervenções práticas.

Nos programas 2 e 3 os conteúdos são direcionados aos principais transtornos psicopatológicos, ensino do rastreio cognitivo e alguns modelos de intervenção para reabilitação neuropsicológica. O programa 4 os módulos estão voltadas apenas para os problemas de aprendizagem. É valido salientar, que os protocolos de reabilitação não são bem definidos nas ementas.

Os programas 5 e 6 contemplam todos os conhecimentos desse domínio, incluindo o processo de avaliação e reabilitação psicológica, incluindo o uso de testes psicológicos, neuropsicológicos e psicopedagógicos. Somente esses programas incluem elaboração de laudos e encaminhamentos.

No programa 7 apresenta os conhecimentos da neuropsicologia, mas a parte da avaliação é direcionada apenas as dificuldades de aprendizagem, memória, linguagem e funções executivas, os instrumentos utilizados estão mais direcionados para a psicopedagogia.

Tabela 2 - Ênfase do Curso.

\begin{tabular}{cl}
\hline Programa & \multicolumn{1}{c}{ Carga Horária } \\
\hline 1 & Educação \\
2 & Avaliação e intervenção \\
3 & Avaliação e intervenção \\
4 & Educação e intervenção \\
5 & Educação, avaliação e intervenção \\
6 & Educação, avaliação e intervenção \\
7 & Educação e avaliação \\
\hline
\end{tabular}




\section{Estágios}

Conforme explicitado na tabela 3 somente o programa 1 - EaD não possui carga horário de estágio supervisionado, todos os outros programas possuem essa carga horária estabelecida em 60 horas distribuídos nos meses do curso.

Tabela 3 - Estágios.

\begin{tabular}{cl}
\hline Programa & \multicolumn{1}{c}{ Carga Horária } \\
\hline 1 & 0 \\
2 & 60 horas \\
3 & 60 horas \\
4 & 60 horas \\
5 & 45 horas de avaliação 15 reabilitação \\
6 & 60 horas \\
7 & 140 horas \\
\hline
\end{tabular}

\section{Público Alvo}

$\mathrm{Na}$ tabela 4 , percebem-se que 5 programas que restringem o público alvo para psicólogos (Programas 2,3,4,6 e 7), o currículo 5 estabelece duas ênfases, sendo que a grade para psicologia tem carga horária maior (80h para o ensino dos instrumentos), avaliação e reabilitação e a segunda grade de formação geral aberta para qualquer pessoa que possua nível superior. Apenas um programa (Número 1) destoa da proposta do conhecimento neuropsicológico, que além de ser um curso somente informativo, abre para qualquer diplomado e ministra avaliação psicológica como módulo.

Tabela 4 - Público Alvo.

\begin{tabular}{cl}
\hline Programa & \multicolumn{1}{c}{ Carga Horária } \\
\hline 1 & Nível superior \\
2 & Psicólogo \\
3 & Psicólogo \\
4 & Psicólogo \\
5 & Psicólogo e formação geral \\
6 & Psicólogo \\
7 & Psicólogo \\
\hline
\end{tabular}

\section{DISCUSSÃO E CONSIDERAÇÕES FINAIS}

A autonomia metodológica, curricular e pedagógica dada as universidade com a resolução $\mathrm{n}^{\circ} 1$ de 8 de junho de 2007, que estabelece normas para o funcionamento dos cursos de pós-graduação Lato Sensu, gera uma dificuldade em ter um processo de formação dentro das especializações de uma maneira mais uniforme, pois independentemente de onde o profissional fizer a sua formação ele obterá o título de Neuropsicólogo, habilitado em todo território nacional. A dúvida que se segue é saber como será essa prática, visto a ampla formação nessa especialidade no país, bem como, a falta de critério em suas ementas e projetos políticos pedagógicos e principalmente em suas ênfases. O que de alguma maneira se faz necessário refletir sobre a autonomia e liberdades das universidades em relação a autonomia e interação da teoria e prática. ${ }^{9}$

O profissional que optou em fazer o curso de
Neuropsicologia e que a sua grade apenas contempla os estudos das neurociências e transtornos mais prevalentes, será habilitado como Neuropsicólogo, mas pode nunca ter visto nada sobre avaliação e muito menos sobre reabilitação. Então como fica esse saber? De que maneira essa profissional, mesmo habilitado atuará em sua prática?

Outro aspecto apontado nessa pesquisa é a oferta da especialidade para qualquer profissional de nível superior, sem o cuidado de olhar as ementas e propostas do curso. Curso de público livre, que oferecem disciplinas de avaliação psicológica, saber esse restrito à psicólogos. Questões que precisam ser refletidas. Como oferecer disciplina de avaliação psicológica para profissional que não poderá atuar na área? Alguns programas oferecerem a disciplina de avaliação neuropsicológica usando apenas testes cognitivos, embora eles possam ser complementares, se a avaliação é neuropsicológica, a testagem é outra.

Construir um currículo de pós-graduação em Neuropsicologia, em que o marco teórico, técnico e científico está apoiado em outra abordagem psicológica não é muito coerente, nem com o programa, nem com o avanço das neuropsicologia. Mesmo sendo uma ciência interdisciplinar, ela tem um direcionamento específico, próprio, que justifica esse novo campo do saber.

Esse estudo também sinalizou a importância de rever o processo de reabilitação/intervenção, que embora seja ofertado na maior parte dos programas, os seus conteúdos não são tão claros. Essa questão se esbarra numa dificuldade da literatura, onde o conhecimento da reabilitação neuropsicológica, ainda ser muito incipiente no Brasil (a maior parte da literatura é em inglês) ela ainda se encontra bem fragmentada, necessitando de um árduo trabalho de seleção e classificação das mesmas. $\mathrm{E}$, mesmo assim, esse conhecimento levantado nos periódicos, ainda não garantem aplicabilidade clínica, pois normalmente esses conhecimento estão fragmentados em muitos artigos e protocolos piloto. Embora o campo da educação já vem trabalhando com a neuropsicologia há tempos, em suas modalidades de ensino, assim como, em suas práticas inclusivas. ${ }^{10}$

Nesse sentido é importante avançar na realização em pesquisas sobre reabilitação neuropsicológica no Brasil $^{11}$, não no sentido de criar um protocolo unificado para as disfunções, mesmo porque, quando fala-se de reabilitação, não está falando apenas da funções específicas, mas uma série de fatores funcionais, orgânicos, sinápticos, que ocorrem conjuntamente dentro de um contexto de subjetividade do indivíduo. Mas, para que o avanço das pesquisas e dos protocolos de intervenção venham contribuir para uma melhor qualidade de vida para quem precisa desses serviços. E também, aprofundar em mais estudos curriculares dessa especialidade no país, para que essas revisões possam sinalizar suprir as carências e incoerências apontadas nesse artigo.

\section{REFERÊNCIAS}

1. Cagnin Simone. A Pesquisa em Neuropsicologia: Desenvolvimento Histórico, Questões Teóricas e Metodológicas. Psicol Pesq 2010;4(2):118-34.

2. Labos E, Slachevsky A. Tratado de neuropsicologia clínica. 
Bolsa de Comercio de Córdoba, 2008.

3. Lezak MD. Neuropsychological assessment. Oxford University Press, USA, 2004.

4. Luria AR. Fundamentos de Neuropsicologia. Rio de Janeiro: Livros Técnicos e Científicos; São Paulo: EDUSP, 1981.

5. Damásio A. O erro de Descartes: emoção, razão e o cérebro humano. Editora Companhia das Letras, 2012.

6. Conselho Federal de Psicologia (2004). Resolução CFP $n^{\circ}$ 2/2004. Brasília: CFP.

7. Brasil. Ministério da Educação. Portaria Normativa $N^{\circ} 01$ 8/07. Avaliação de cursos, 12 jan. 2007.

8. Gil AC. Como elaborar projetos de pesquisa. 4ed. São Paulo, Atlas, 2002.
9. Fonseca M, Fonseca DM. A gestão acadêmica da pósgraduação lato sensu: o papel do coordenador para a qualidade dos cursos. Educação e Pesquisa 2016;42(1):15164. doi: 10.1590/S1517-9702201603136263.

10. Galvani Claúdia. Psicomotricidade colaborando com a formação de educadores no processo de inclusão: 0 corpo como elemento fundamental na estrutura da aprendizagem. Plures Humanidades 2017;18(1):65-78.

11. Ramos AA, Hamdan AC. O crescimento da avaliação neuropsicológica no Brasil: uma revisão sistemática. Psicologia: Ciência e Profissão 2016;36(2):471-85. doi: 10.1590/1982-3703001792013

Como citar: PEREIRA, Diogo Fagundes. Neuropsicologia, formação e desafios. Cinergis, Santa Cruz do Sul, v. 18, n. 4, out. 2017. ISSN 2177-4005. Disponível em: <https://online.unisc.br/seer/index.php/cinergis/article/view/9487>. Acesso em: 06 dez. 2017. doi:http://dx.doi.org/10.17058/cinergis.v18i4.9487. 